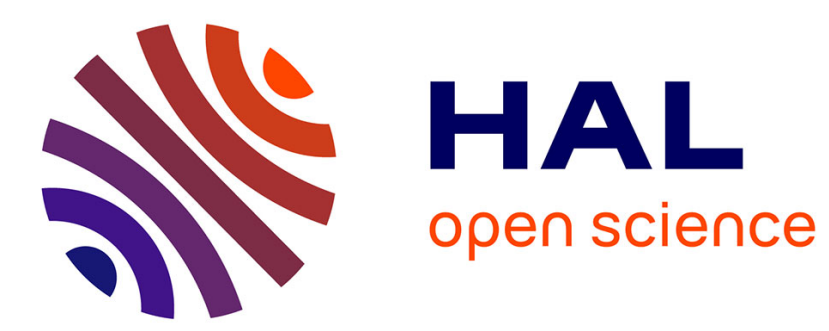

\title{
A Semi-empirical Model of Test Quality in Symmetric Testing: Application to Testing Java Card APIs
}

Arnaud Gotlieb, Patrick Bernard

\section{To cite this version:}

Arnaud Gotlieb, Patrick Bernard. A Semi-empirical Model of Test Quality in Symmetric Testing: Application to Testing Java Card APIs. [Research Report] RR-5675, INRIA. 2005, pp.21. inria00070338

\section{HAL Id: inria-00070338 \\ https://hal.inria.fr/inria-00070338}

Submitted on 19 May 2006

HAL is a multi-disciplinary open access archive for the deposit and dissemination of scientific research documents, whether they are published or not. The documents may come from teaching and research institutions in France or abroad, or from public or private research centers.
L'archive ouverte pluridisciplinaire HAL, est destinée au dépôt et à la diffusion de documents scientifiques de niveau recherche, publiés ou non, émanant des établissements d'enseignement et de recherche français ou étrangers, des laboratoires publics ou privés. 
INSTITUT NATIONAL DE RECHERCHE EN INFORMATIQUE ET EN AUTOMATIQUE

\section{A Semi-empirical Model of Test Quality in Symmetric Testing: Application to Testing Java Card APIs}

Arnaud Gotlieb , Patrick Bernard

\section{$\mathbf{N}^{\circ} 5675$}

September 2005

Systèmes cognitifs 



\title{
RINRIA
}

\section{A Semi-empirical Model of Test Quality in Symmetric Testing: Application to Testing Java Card APIs}

\author{
Arnaud Gotlieb* ${ }^{*}$ Patrick Bernard ${ }^{\dagger}$ \\ Systèmes cognitifs \\ Projet Lande \\ Rapport de recherche $\mathrm{n}^{\circ} 5675$ - September 2005 - 21 pages
}

\begin{abstract}
In the smart card quality assurance field, Software Testing is the privileged way of increasing the confidence level in the implementation correctness. Software testing involves a set of activities that includes at least test data selection, test execution and output correctness checking. When testing Java Card application programming interfaces (APIs), the tester has to deal with the classical so-called oracle problem, i.e. to find a way to evaluate the correctness of the computed output. In this paper, we report on an experience in testing methods of the Oberthur Card Systems Cosmo 32 RSA V3.4 Java Card APIs by using the Symmetric Testing paradigm. This paradigm aims at using user-defined symmetry properties of methods as test oracles. We propose an experimental environment that combines random testing and symmetry checking for on-card testing of several symmetric methods of Java Card APIs. Based on this environment, we develop a semi-empirical model (a model feeded by experimental data) to help deciding when to stop testing and to assess test quality. First experimental results are reported and the extension of the approach to non-symmetric Java Card API methods is discussed.
\end{abstract}

Key-words: Program Testing, Test quality, Symmetric Testing, Java Card

(Résumé : tsvp)

* Projet Lande - IRISA / INRIA - Campus de Beaulieu, 35042 Rennes Cedex, FRANCE

$\dagger$ OBERTHUR CARD SYSTEMS, rue Auguste Blanche, 92800 PUTEAUX, FRANCE 


\section{Un modèle semi-empirique de la qualité de test en test symétrique : application au test d'interfaces Java Card}

Résumé : Dans le domaine de l'Assurance Qualité des cartes à puce, le test logiciel est le moyen privilégié permettant d'augmenter la confiance que nous portons dans la correction d'une implantation. Le test logiciel comporte un ensemble de tâches contenant au moins la séléction des données de test, l'éxécution des tests et le contrôle de la correction des sorties calculées. Lorsque les interfaces de la plateforme Java Card sont testées, le testeur doit faire face au classique problème de l'oracle, c'est à dire qu'il doit trouver un moyen d'evaluer la correction des sorties calculées. Dans ce papier, nous rapportons une expérience dans laquelle nous avons utilisé le paradigme du test symétrique pour tester des interfaces de la plateforme Java Card Oberthur Card Systems Cosmo 32 RSA V3.4. Ce paradigme consiste à utiliser des propriétés de symétrie données par l'utilisateur comme oracles de test pour les interfaces. Nous proposons un environnement expérimental qui combine test aléatoire et contrôle de symétrie pour automatiser le test de méthodes d'interfaces Java Card. Basé sur cet environnement, nous avons développé un modèle semi-empirique (un modèle nourri par des données expérimentales) pour aider à décider du moment opportun de l'arrêt des tests et évaluer la qualité du test. Les premiers résultats expérimentaux sont donnés et l'extension de cette approche aux méthodes non-symétriques est discutée.

Mots-clé : Test Logiciel, Qualité de test, Test symétrique, Java Card 


\section{Introduction}

Although formal verification and software testing were viewed as opposites for a long time, with formal verification concentrating on proving program correctness while testing concentrating on finding faults in program implementation, they can now be considered as complementary techniques [4]. In the smart card field, software testing is required by the Common Criteria evaluation scheme [20] to increase the confidence level of the certifying authority in the implementation correctness of security functions. In this context, techniques and tools that permit to automate (even partially) the testing process are welcome. Early research works in that field include the BZ-testing approach designed by Legeard et al. $[21,1]$ to generate automatically test cases from a formal $\mathrm{B}$ or $\mathrm{Z}$ specification. The corresponding tools suite has been employed to validate the Java Card transaction mechanism by generating test cases on the boundary states of the formal specification [22]. In [29], Pretschner et al. followed a similar approach by using the AUTOFOCUS tool for specifying the command/response mechanism of an inhouse smart card and generating test cases for validating the authentification protocol of the card. At the same time, Clarke et al. [9] developed symbolic test generation algorithms and applied them to generate on-the-fly test cases for a feature of the CEPS ${ }^{1}$ e-purse application and Martin and Du Bousquet [24] proposed to use UML-based tools to generate test suites for testing Java Card applets.

All these approaches have in common to require first a formal model ( $\mathrm{Z}$ or $\mathrm{B}$ specification, automata, input/output transition system or statecharts) to be constructed in order to generate test cases. When the time-to-market of a new product is critical, this effort appears as being too costly and cheaper (but still rigorous) approaches are needed. Techniques such as statistical testing [13, 31], boundary testing [11], or local exhaustive testing [33] do not require a formal model to be developed. Statistical testing aims at selecting randomly the values inside the input domain of the application under test by using pseudo-random numbers generators, boundary testing relies on selecting the boundaries of an input space partition, whereas local exhaustive testing systematically explores a bounded part of the input domain. In these approaches, testing just depends on the availability of oracles, that is, some procedures for predicting the expected results of the applications under test. Unfortunately, as earlier pointed out by Weyuker [32], there are programs to be tested for which the design of oracles is a non-trivial task. Examples of such programs in the smart card field include standard and proprietary Java Card APIs as they are just usually described by their interfaces and a few lines of natural text ${ }^{2}$. For these APIs, current industrial practices rely on coding the oracle as the result of another program that will be confronted with the result of the API under test. This approach suffers from several drawbacks such as the high cost of the development of oracles and the existence of faults into the oracles.

Recently, we have proposed [16] to address this oracle problem for Java programs by using user-defined symmetries of programs to check the correctness of the computed output. Here, symmetries are input-output permutation relations over program executions that lead

\footnotetext{
${ }^{1}$ The Common Electronic Purse Specification is a standard for creating inter-operable multi-currency smart card e-purse systems.

${ }^{2}$ Although formalizations do exist [25].
} 
to partitioning the input space into equivalence classes and the equivalence between two executions serves as an oracle. We introduce a software testing paradigm called Symmetric Testing, where automatic test data generation was coupled with symmetries checking and local exhaustive testing to uncover faults inside the programs.

In this paper, we report on an experience in applying Symmetric Testing to test methods of the Oberthur Card Systems Cosmo 32 RSA V3.4 Java Card API [27] by using random testing. Unlike our previous work [16], we develop here an original semi-empirical model to help decide when to stop testing and to assess test quality in Symmetric Testing. This model is feeded with an empirical parameter (based on symmetry checking) in a theoretical model of random testing, in order to obtain the minimum number of test data required to reach a given level of quality. From the Oberthur Card Systems Cosmo 32 RSA V3.4 Java Card API [27], we have selected the methods to test by studying their symmetry properties, as Symmetric Testing is only suitable for testing programs that possesses input-output symmetry relation. By using several tools, we have designed an experimental environment to build our semiempirical model and to apply Symmetric Testing in situations as close as possible to the real situations. In contrast with other research works in testing Java card programs [9, 29], test execution and symmetries checking have been conducted by cross-testing on a smart card and not by using simulations.

The rest of the paper is organized as follows: section 2 presents the Symmetric Testing paradigm and gives examples of symmetry relations. Section 3 reports the symmetry analysis of a few methods of the Oberthur Card Systems Cosmo 32 RSA V3.4 Java Card API while section 4 details our semi-empirical model of random testing based on symmetries checking. Section 5 reports the first experimental results and discusses extension of the framework to handle non-symmetric methods of the Java Card APIs. Finally section 6 pinpoints several perspectives to this work.

\section{Symmetric Testing}

Exploiting symmetry in verification is not a new idea. Emerson and Sistla [14] and Ip and Dill [19] proposed early to exploit structural symmetries to address the problem of state explosion in model checking. This approach has been experienced and proved interesting in practice in several tools, such as VeriSoft [15] or SPIN [3]; its principle is based on basic results from group theory [14, 19, 15] and partial order techniques [10].

Based on similar ideas, we recently introduced Symmetric Testing [16] in the context of Software Testing. The flavour of our approach is explained here on a very basic example. Consider a program $P$ intended to compute the greatest common divisor $(g c d)$ of two nonnegative integers $u$ and $v$ and suppose that $P$ is tested with the following test datum $(u=$ $1309, v=693$ ) automatically generated by a random test data generator. Although we all know how to compute the gcd of two integers ${ }^{3}$, it is not so easy to predict the expected value of $\operatorname{gcd}(1309,693)$ without the help of a calculator. Fortunately, $g c d$ satisfies a simple

\footnotetext{
${ }^{3}$ With the Euclidian algorithm for example.
} 
symmetry relation: $\forall u \forall v, g c d(u, v)=g c d(v, u)$. So, if $P(1309,693) \neq P(693,1309)$ then the testing process will succeed to uncover a fault in $P$ without the help of a complete oracle of $g c d$. Note that such a symmetry relation is a necessary but not sufficient condition, for the correctness of $P$.

Identifying such symmetry relations for larger programs might appear to be difficult or useless to detect non-trivial fault. On the contrary, we argue that numerous programs have to satisfy symmetry relations and these relations are useful for detecting subtle faults. In fact, every program $P$ that takes an unordered set as argument has to satisfy a symmetry relation: the expected outcome of $P$ is invariant under any permutation of the elements of the set. Numerous programs take unordered sets as arguments: consider sorting or selection programs that are used in search engines, programs that operate over data buffers, or graph-based programs just to name a few. Note that experimental evidence were provided to support this argument $[8,6,16]$.

\subsection{Symmetry relations}

We generalized the above idea to obtain a formal and generic definition of symmetry relation. This definition is based on basic results from Group theory that are briefly recalled here. A detailed but still accessible presentation can be found in [2].

The notion of symmetric group is the corner-stone of Symmetric Testing. The symmetric group $S_{n}$ is the set of bijective mappings from $\{1, . ., n\}$ to itself. It has exactly $n$ ! elements, called permutations. A permutation in $S_{n}$ is written: $\theta=\left(\begin{array}{ccc}1 & . . & n \\ i(1) & . . & i(n)\end{array}\right)$ where $i(1), . ., i(n)$ denote the images of $1, . ., n$ by the permutation $\theta$. A group action of $S_{n}$ on a set $X$ is a mapping $(\theta, x) \mapsto \theta \cdot x$ such as: $i d_{S_{n}} \cdot x=x$ and $\left(\theta_{1} \circ \theta_{2}\right) \cdot x=\theta_{1} \cdot\left(\theta_{2} \cdot x\right)$ for all $x \in X$ and $\theta, \theta_{1}, \theta_{2} \in S_{n}$ (we say that $S_{n}$ acts on $E$ and $\theta$ acts on $x$ ). Note that $X$ is closed under the action of $S_{n}$.

It is well-known that any permutation can be expressed as the composition of certain simple permutations, called cycles. Consider for example the permutation

$\theta=\left(\begin{array}{lllll}1 & 2 & 3 & 4 & 5 \\ 3 & 4 & 1 & 5 & 2\end{array}\right)$ of $S_{5}$, the same permutation can be written as $\theta=\left(\begin{array}{llll}1 & 3\end{array}\right)\left(\begin{array}{lll}2 & 4 & 5\end{array}\right)$ where each pair of brackets denotes a cycle $\left(a_{1} a_{2} \ldots a_{r}\right)$, that maps $a_{1}$ to $a_{2}, . . a_{r-1}$ to $a_{r}$, $a_{r}$ to $a_{1}$ and leave unchanged the other elements. A cycle written $\left(a_{i} a_{j}\right)$ is usually referred to as a transposition.

A subset $X$ of elements of a symmetric group $S_{n}$ is a set of generators iff every element of $S_{n}$ can be written as a finite composition of the elements of $X$. For example, $S_{3}$ is generated by the two transpositions $\tau_{1}=\left(\begin{array}{ll}1 & 2\end{array}\right)$ and $\tau_{2}=\left(\begin{array}{ll}2 & 3\end{array}\right)$. More generally, $S_{n}$ is generated by the transposition $\tau=\left(\begin{array}{ll}1 & 2\end{array}\right)$ and the cycle $\sigma=\left(\begin{array}{lll}1 & 2 & \ldots\end{array}\right)$ and cannot be generated by less than two permutations [2].

Symmetries of the function computed by a program $P$ become interesting with regards to testing when they express general abstract properties. This leads to the notion of symmetry relations for a program.

$\mathrm{RR} \mathrm{n}^{\circ} 5675$ 
Definition 1 (symmetry relation) Let $P$ be a program that computes a function $f$ over an input domain dom $(f)$ toward a range domain ran $(f)$, and let $S_{n}$ act on dom $(f)$ with a group action . and $S_{m}$ act on ran $(f)$ with a group action $\odot$. A symmetry relation $\Psi_{n, m}$ holds for $P$ iff

1. $\forall \theta \in S_{n}, \exists \eta \in S_{m}$ such that $\forall x \in \operatorname{dom}(f), f(\theta \cdot x)=\eta \odot f(x)$

2. $\Psi_{n, m}: \theta \longmapsto \eta$ is a homomorphism from $S_{n}$ to $S_{m}$

The first item requires $f$ to satisfy an invariant property for all $\theta$ in $S_{n}$ and for all $x$ in the input domain of $f$. Note that $\eta$, the image of $\theta$ by the symmetry relation $\Psi_{n, m}$, is independent of the choice of $x$. Most of the time the two group actions will be the same $(\cdot \equiv$ $\odot)$, however we will see below an example of distinct group actions in a symmetry relation. The second item requires the symmetry relation to be a homomorphism. A homomorphism is a map $\varphi$ from $G_{1}$ to $G_{2}$ such that $\varphi\left(\theta \circ \theta^{\prime}\right)=\varphi(\theta) \circ \varphi\left(\theta^{\prime}\right)$ for all $\theta, \theta^{\prime} \in G_{1}$. Informally speaking, this requirement guarantees the symmetric structure of $\operatorname{dom}(f)$ to be preserved by application of $f$, allowing so nice composition properties of symmetric relations. In our framework, we make an extensive use of this property to optimize the symmetry testing process, as explained below.

\subsection{Examples}

As an example, consider the Java Card program void max3(byte[] A, byte[] B) which selects the three maximum values of the array $A$ and sorts them into the array $B$. If $n$ denotes the size of $A(n \geq 3)$ and $f$ denotes the function computed by max3 from $\mathbb{B}^{n}$ to $\mathbb{B}^{3}$, where $\mathbb{B}$ is the finite set of all possible bytes whose values are 8-bit signed two's complement integers, then the program max3 has to satisfy a $\Psi_{n, 3}$ symmetry relation because the array $\mathrm{B}$ is invariant under any permutation of $\mathrm{A}$. Here, the considered group action (in both cases) is defined by: $S_{n} \times \mathbb{B}^{n} \rightarrow \mathbb{B}^{n},\left(\theta, A=\left[a_{1}, . ., a_{n}\right]\right) \mapsto \theta \cdot A=\left[a_{\theta^{-1}(1)}, . ., a_{\theta^{-1}(n)}\right]$

As a more complicated example, consider a Java Card program short get Index 0 (short [] A) that takes an array A of (non-negative) distinct values as argument and returns the index of the occurrence of 0 in the array or throws an exception if 0 is not present in $A$. The program getIndex 0 computes a function $f$ from $\mathbb{S}^{n}$ to $\{0,1, . ., n-1\} \cup\{$ err $\}$ where $\mathbb{S}$ denotes the finite set of 32-bit signed short integers, $n$ denotes the size of A and err denotes an erroneous symbolic value. When 0 belongs to the array A, getIndex0 has to satisfy a $\Psi_{n, n}$ symmetry relation because, 1$)$ for any $\theta \in S_{n}, f(\theta \cdot A)=\theta \odot f(A)$ for all $A \in \mathbb{S}^{n}$ that contains an occurrence of 0 , and 2) the identical map $\theta \longmapsto \theta$ is a group homomorphism. For instance, if $A=[98,4578,1258,654,2589,558,12577,0,4876,25541]$ and $\theta=\left(\begin{array}{lll}1 & 2\end{array} .10\right)$ then $f(A)$ returns 7 and $f(\theta \cdot A)$ returns 8 which is the image of 7 by $\theta$ when it acts over $\{0,1, . ., 9\}$. Note that this example shows two distinct group actions: $S_{n}$ acts over $\mathbb{S}^{10}$ when it is applied to the input sequence $A$ of $f$ whereas it acts over $\{0,1, . ., 9\}$ when applied to the outcome of $f$ with the following group action: 
$S_{n} \times\{0,1, . ., n-1\} \rightarrow\{0,1, . ., n-1\},(\theta, x) \mapsto \theta \odot y=\theta(x)$

\subsection{Symmetric Testing}

Symmetry relations can be used to seek for a subclass of faults within an implementation. Informally speaking, the Symmetric Testing principle aims at finding counter-examples (called symmetry violations) of the symmetry relation that a program has to satisfy.

Definition 2 (Symmetry violation) let $P$ be a program over an input domain $D$ and $\Psi_{k, l}$ be a symmetry relation that $P$ has to satisfy, then a symmetry violation for $P$ w.r.t. $\Psi_{k, l}$ is a couple $(x, \theta)$ such as $x \in D, \theta \in S_{n}$ and $P(\theta \cdot x) \neq \Psi_{k, l}(\theta) \odot P(x)$.

The interesting point here is that symmetry violation can be checked by program executions whereas trying to prove formally that the function computed by a program satisfies a symmetry relation would require to express semantics of the language. Note that there is no way to identify among the two test data $x$ and $\theta \cdot x$ the one that leads to an incorrect outcome for $P$. In the worst case, they can even be both faulty. So, given a set of test data and a symmetry relation, we get a naive procedure that can uncover a subclass of faults in $P$ : it requires to compute $P$ with all the permutations of the permutable inputs of each vector $x$ in the test set and then to check whether the outcome vectors are equal to a permutation of the vector returned by $P$. The latter operation is called an outcome comparison.

However, the somehow naive procedure given above requires an outcome comparison for each possible permutation in the Symmetric Group $S_{n}$ and, as $S_{n}$ contains $n$ ! permutations, the approach becomes impractical when $n$ increases. The following result is of help to reduce the number of outcome comparisons and to provide a more interesting approach.

Theorem 1 Let $P$ be a program that computes a function $f$ and $\Psi_{k, l}$ be a symmetry relation for $P$, let $\tau=\left(\begin{array}{ll}1 & 2\end{array}\right)$ and $\sigma=\left(\begin{array}{lll}1 & 2 & . . k\end{array}\right)$, then we have

$$
\left\{\begin{array}{l}
f \circ \tau=\Psi_{k, l}(\tau) \circ f \\
f \circ \sigma=\Psi_{k, l}(\sigma) \circ f
\end{array} \quad \Longleftrightarrow f \circ \theta=\Psi_{k, l}(\theta) \circ f \quad \forall \theta \in S_{k}\right.
$$

The proof of this theorem can be found in [16]; it is based on the fact that $\Psi_{k, l}$ is required to be a group homomorphism. Hence, by showing that $f(\tau \cdot x)=\Psi_{k, l}(\tau) \odot f(x)$ and $f(\sigma \cdot x)=\Psi_{k, l}(\sigma) \odot f(x)$, we get $f(\theta \cdot x)=\Psi_{k, l}(\theta) \odot f(x)$ for all $\theta \in S_{k}$, meaning that only two permutations are required to be checked in the above procedure. Moreover, by noticing that if $(x, \theta)$ is a symmetry violation then $\left(\theta \cdot x, \theta^{-1}\right)$ is automatically another symmetry violation, we can again restrict the input domain to explore.

The rest of the paper reports on our experience in applying Symmetric Testing in the context of APIs Java Card testing. 


\section{$3 \quad$ Symmetry in Java Card API}

Unlike other smart cards, a Java Card includes a Java Virtual Machine and a set of API classes implemented in its read-only memory part. The Java Card Virtual Machine provides the interpretation of Java Card language constructs and the APIs are a set of classes and interfaces providing additional functionality that can be accessed by Java Card applets. A complete view of the development process of Java Card applets can be found in [18]. The OCS $^{4}$ Cosmo 32 RSA V3.4 (called Cosmo in the following) contains an implementation of the Java Card APIs.

\subsection{The Cosmo Java Card APIs}

The structure of the Cosmo Java Card platform is given in Fig.1. It consists of several components, such as an implementation of the Java Virtual Machine, the open platform applications, a set of packages implementing the standard SUN's Java Card API [30] and a set of proprietary packages. The four OCS proprietary packages consists of standard security services such as the VISA Open Platform Provider Security Domain, a set of base classes for implementing a Provider Security Domain, a complete range of classes for creating, maintaining and inspecting the card file-system and methods that are useful for JCRE related operations. Note that the Cosmo Java Card platform includes garbage collection facilities.

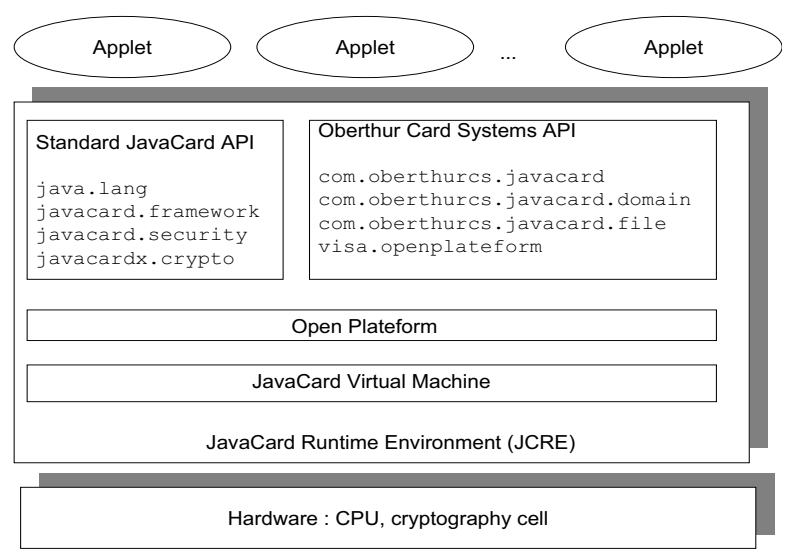

Figure 1: The OCS Cosmo 32 RSA V3.4 platform

\footnotetext{
${ }^{4}$ Stands for Oberthur Card Systems
} 


\subsection{Symmetry analysis of a selected Cosmo Java Card API class}

Among the possibilities, we selected

com.oberthurcs.javacard.file.Utilfs as a case study because it consists of several generic utility methods that present symmetries. All the methods operate on byte or object arrays and are useful for dealing with APDU buffers. The Utilfs class is composed of 10 methods that are shown in Tab.1 together with their symmetry relations. The first and second column are extracted from the Cosmo API informal specification [27]. The third column summarizes the results of our symmetry analysis. The set of all possible bytes is noted $\mathbb{B}$ and the set of available objects is noted $\mathbb{O}$. Note first that some methods that deal with multiple array elements are tagged as NonAtomic. Atomicity defines how the card handles the contents of persistent storage after a failure or fatal exception during an update of a class field or an array component [30]. An applet might not require atomicity for array updates. The Utilfs.arrayAndNonAtomic method is an example: it shall not use the transaction commit buffer even when called with a transaction in progress.

Among the ten methods of this class, we found that seven have to satisfy a simple symmetry relation. We discuss a few of them; the other ones can easily be deduced from these. The method short arrayAndNonAtomic ( byte [] dest, short dest0ff, byte [] src, short src0ff, short len) can be abstracted by a function $f$ from $\mathbb{B}^{\text {len }} \times \mathbb{B}^{\text {len }}$ to $\mathbb{B}^{l e n}$ as it modifies the input-output parameter dest by combining src and dest and by considering all other parameters as non-variable. arrayAndNonAtomic has to satisfy a $\Psi_{l e n, l e n}$ symmetry relation because of the following invariant property:

$$
\forall \theta \in S_{l e n}, f(\theta \cdot \text { dest }, \theta \cdot s r c)=\theta \odot f(\text { dest }, \text { src })
$$

This is due to the fact that dest and src represent two unordered sets of values for this method. Note that the two group actions are distinct as the first one holds over the set $\mathbb{B}^{l e n} \times \mathbb{B}^{\text {len }}$ (i.e. $\theta \cdot(x, y)=(\theta \cdot x, \theta \cdot y)$ where $x$ and $y$ are vectors of $\mathbb{B}^{\text {len }}$ ) whereas the second one $\odot$ holds just over $\mathbb{B}^{l e n}$. The methods short arrayCompare( byte[] src, short src0ff, byte patByte, short length) and short arrayFindByte( byte[] src, short src0ff, short len, byte pattern) have each to satisfy a $\Psi_{l e n, l e n}$ symmetry relation as the following invariant property holds: $\forall \theta \in S_{l e n}, f(\theta \cdot s r c)=\theta \odot f(s r c)$ where $f$ denotes a map from $\mathbb{B}^{l e n}$ to $\{1, . ., l e n\}$. In fact, these two programs are selection programs that are invariant to permutations of a subset of their input parameters. In the Utilfs class, some methods do not have to satisfy simple symmetry relations. For example, the method arrayFindShort has incompatible input types, that is to say the method looks for a short integer variable into an array of bytes. Although we have not realized a full study of the Cosmo Java Card APIs, we took a look at other classes to find symmetry relations. For example, the classes visa.openplatform. OPSystem, javacard. security. MessageDigest or javacard.framework. Util contain methods that have to satisfy symmetry relations. Note that the compositional definition of symmetry relations allows to combine several method calls. However, we also found numerous classes where the symmetry analysis does not reveal any symmetry relations. Examples of such classes include com. oberthurcs.javacard.file.* or javacard.framework. JCSystem. So, the Symmetric Testing approach remains limited 
Table 1: Symmetry in the OCS Utilfs methods

\begin{tabular}{|c|c|c|}
\hline $\begin{array}{l}\text { Signature of Java } \\
\text { Card methods }\end{array}$ & Informal specifications & Symmetry relations \\
\hline $\begin{array}{l}\text { short } \\
\text { arrayAndNonAtomic( } \\
\text { byte[] dest, short } \\
\text { dest0ff, byte [] } \\
\text { src, short src0ff, } \\
\text { short len) }\end{array}$ & $\begin{array}{l}\text { Copies the result of a bit- } \\
\text { wise AND on the first } \\
\text { operand dest and the second } \\
\text { operand src into dest }\end{array}$ & $\begin{array}{l}\text { The program computes the following function: } \\
f: \mathbb{B}^{\text {len }} \times \mathbb{B}^{\text {len }} \longrightarrow \mathbb{B}^{\text {len }} \\
\quad(\text { dest }, \text { src }) \longmapsto \text { dest }^{\prime} \\
\text { where dest } \text { stands for the value dest computed } \\
\text { after application of the arrayAndNonAtomic program. } \\
\text { It has to satisfy a } \Psi_{\text {len, } \text { len }} \text { symmetry relation. }\end{array}$ \\
\hline $\begin{array}{l}\text { short arrayCompare( } \\
\text { byte [] src, short } \\
\text { src0ff, byte } \\
\text { patByte, short } \\
\text { len) }\end{array}$ & $\begin{array}{l}\text { Returns the index of the } \\
\text { first byte in the specified } \\
\text { part of src that does not } \\
\text { match patByte, or 0xFFFF } \\
\text { if every byte matches }\end{array}$ & 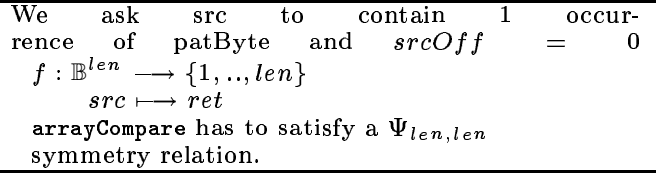 \\
\hline $\begin{array}{l}\text { short } \\
\text { arrayFindByte ( } \\
\text { byte [] src, short } \\
\text { src0ff, short len, } \\
\text { byte pattern) }\end{array}$ & $\begin{array}{l}\text { Returns the index of the } \\
\text { first byte in the part of sr- } \\
\text { c that matches the specified } \\
\text { pattern. }\end{array}$ & $\begin{array}{l}\text { We ask src to contain } 1 \text { occurrence of pattern } \\
f: \mathbb{B}^{l e n} \longrightarrow\{1, \ldots \text {, len }\} \\
\quad \text { src } \longmapsto \text { ret } \\
\text { arrayFindByte has to satisfy a } \Psi_{l e n, l e n} \\
\text { symmetry relation. }\end{array}$ \\
\hline $\begin{array}{l}\text { short } \\
\text { arrayFindPattern( } \\
\text { byte [] src, short } \\
\text { src0ff, short } \\
\text { srcLen, byte [] } \\
\text { patSrc, short } \\
\text { patOff, short } \\
\text { patLen) }\end{array}$ & $\begin{array}{l}\text { Returns the index of the } \\
\text { first byte in the part of sr- } \\
\text { c that matches the specified } \\
\text { pattern. }\end{array}$ & no simple symmetry \\
\hline $\begin{array}{l}\text { short } \\
\text { arrayFindShort ( } \\
\text { byte [] src, short } \\
\text { src0ff, short len, } \\
\text { short pattern) }\end{array}$ & $\begin{array}{l}\text { Returns the index of the } \\
\text { first byte in the part of sr- } \\
\text { c that matches the specified } \\
\text { pattern (a short is } 2 \text { bytes). }\end{array}$ & no simple symmetry \\
\hline $\begin{array}{l}\text { short } \\
\text { array0rNonAtomic( } \\
\text { byte[] dest, short } \\
\text { dest0ff, byte [] } \\
\text { src, short src0ff, } \\
\text { short len) }\end{array}$ & $\begin{array}{l}\text { Copies the result of a bit- } \\
\text { wise OR on the first and } \\
\text { second operands into dest. }\end{array}$ & $\begin{array}{l}f: \mathbb{B}^{\text {len }} \times \mathbb{B}^{\text {len }} \longrightarrow \mathbb{B}^{\text {len }} \\
\quad(\text { dest } \text { src }) \longmapsto \text { dest } \\
\text { array0rNonAtomic has to satisfy a } \Psi_{l e n, l e n} \\
\text { symmetry relation. }\end{array}$ \\
\hline $\begin{array}{l}\text { short } \\
\text { arrayXorNonAtomic( } \\
\text { byte[] dest, short } \\
\text { dest0ff, byte [] } \\
\text { src, short src0ff, } \\
\text { short len) }\end{array}$ & $\begin{array}{l}\text { Copies the result of a bit- } \\
\text { wise XOR on the first and } \\
\text { second operands into dest. }\end{array}$ & $\begin{array}{l}f: \mathbb{B}^{l e n} \times \mathbb{B}^{\text {len }} \longrightarrow \mathbb{B}^{\text {len }} \\
\quad(\text { dest }, \text { src }) \longmapsto \text { dest } \\
\text { arrayXorNonAtomic has to satisfy a } \Psi_{l e n, l e n} \\
\text { symmetry relation. }\end{array}$ \\
\hline $\begin{array}{l}\text { short } \\
\text { getObjectIndex ( } \\
\text { java. lang. Object }[] \\
\text { src, short } \\
\text { srcOff, short } n, \\
\text { java.lang.Object } \\
\text { pattern) }\end{array}$ & $\begin{array}{l}\text { Returns the index of the nth } \\
\text { occurrence in the part of src } \\
\text { that matches pattern. }\end{array}$ & $\begin{array}{l}\text { len denotes the length of src and } n \underset{1}{=} \\
\text { we ask src to contain } 1 \text { occurrence of pattern } \\
f: \mathbb{O}^{\text {len }} \longrightarrow\{1, \ldots \text {, len }\} \\
\text { src } \longrightarrow \text { ret } \\
\text { getObjectIndex has to satisfy a } \Psi_{\text {len,len }} \\
\text { symmetry relation. }\end{array}$ \\
\hline $\begin{array}{l}\text { short } \\
\text { getShortIndex ( } \\
\text { short [] src, short } \\
\text { src0ff, short } n, \\
\text { short pattern) }\end{array}$ & $\begin{array}{l}\text { Returns the index of the nth } \\
\text { occurrence in the part of src } \\
\text { that matches pattern. }\end{array}$ & $\begin{array}{l}\text { len denotes the length of src and } n \underset{1}{=} \\
\text { we ask src to contain } 1 \text { occurrence of pattern } \\
f: \mathbb{O}^{l e n} \longrightarrow\{1, \ldots, \text { len }\} \\
\text { src } \longmapsto \text { ret } \\
\text { getShortIndex has to satisfy a } \Psi_{l e n, l e n} \\
\text { symmetry relation. }\end{array}$ \\
\hline $\begin{array}{l}\text { short } \\
\text { getTaggedShort ( } \\
\text { byte[] src, short } \\
\text { src0ff, short } \\
\text { srcLen, short tag) }\end{array}$ & $\begin{array}{l}\text { Returns the index of the } \\
\text { first TLV tag in the part of } \\
\text { src that matches the speci- } \\
\text { fied tag. }\end{array}$ & no simple symmetry \\
\hline
\end{tabular}


in application to a restricted part of the Cosmo Java Card APIs. For these classes and methods, other input-output properties should be taken as partial oracles as discussed in section 5.3.

In order to asses the test quality achieved by the Symmetric Testing approach, we use a semi-empirical model that is now described.

\section{A semi-empirical model}

In the work presented in this paper, the Symmetric Testing principle combines random test data generation and automatic symmetry checking. Random testing has traditionally been viewed as a blind approach of program testing. However, results of actual random testing experiments confirmed its potential to reveal faults and as a validation tool [13]. Nevertheless, when the tester wants to exploit a random test data generator, he faces two main difficulties. The first is the classical oracle problem already discussed in the introduction of this paper: an automatic way of checking the output correctness is required when one wants to use a random test data generator. The second problem is to determine the test quality level reached by a random testing approach. In general, it is difficult to quantify how reliable is a program that has only been tested by randomly generated test data. Several works deal with this problem by using a purely theoretical framework based on probabilistic analysis $[31,23]$. In the work reported here, we make use of a semi-empirical model to help decide when to stop testing, that is to say the use of empirical parameters (based on symmetries checking) in a model that links the test quality to the number of test data randomly generated. This section is devoted to this semi-empirical model.

\subsection{A theoretical model of random testing}

When using a random test data generator, a theoretical model has to be developed to evaluate the effectiveness of this testing method for finding specific classes of faults [23].

Let $p_{f}$ be the probability that a randomly generated input test datum $x$ exhibit a fault in the program $P$. A fault in $P$ can be understood as a syntactical change in the source code that leads, for some input data, to a difference between $P(x)$ and the expected output of the function computed by $P$ with $x$. By a simple probabilistic reasoning, we can develop a model of random testing based on $p_{f}$, that is a law between the number $N$ of randomly generated test data and a probabilistic parameter that characterizes the random testing efficiency $[13,26]$. This parameter is usually referred to as the test quality and it is noted $Q_{N}$ [31]. Formally speaking, $Q_{N}$ is the probability that a set of $N$ randomly generated test data reveals at least one fault in $P$. Hence, a theoretical model of random testing is given by the following relation, for which a detailed explanation can be found in [31]:

Theorem $2\left(1-p_{f}\right)^{N}=1-Q_{N}$

The relation can easily be justified since $\left(1-p_{f}\right)$ is the probability of no fault per execution, and $\left(1-Q_{N}\right)$ is the probability of no detection during $N$ executions. As an 
immediate corollary, we get an estimation of the minimum number of test data to reach a certain value of the test quality $Q_{N}$ :

Corollary $1 N \geq\left\lfloor\frac{\ln \left(1-Q_{N}\right)}{\ln \left(1-p_{f}\right)}\right\rfloor$

where $\lfloor x\rfloor$ denotes the integral part of a real number $x$.

\subsection{The empirical parameter $p_{s}$}

The above theoretical model of random testing suffers from one drawback: it is based on $p_{f}$ which is almost impossible to evaluate without a precise knowledge of all the existing faults in the program $P$, which is exactly the ideal goal of testing. Unlike other authors, we address this problem by using an empirical parameter in place of $p_{f}$ to build our model. This parameter $p_{s}$ is related to symmetry checking: $p_{s}$ is the probability that a test datum $x$ randomly generated in a subset of size $s$ of the input domain would lead to a symmetry violation $(x, \theta)$ for $P$ w.r.t. a given symmetry relation. This parameter characterizes the probability for Symmetric Testing to reveal a fault in $P$ when it makes use of a random test data generator over the input domain.

We propose to empirically evaluate $p_{s}$ on a selected specimen program, by executing several faulty versions of this program. Using a specimen program is advantageous as we can easily build faulty versions of this program by using its source code. The key point of our approach concerns the knowledge of faults for the specimen program and the number of symmetry violations occurring when checking the output correctness of this program. This approach is based on a uniformity hypothesis: the inferred value for the specimen program applies to other programs as well. This hypothesis is debatable and relates to the difficulty of finding a representative sample in Statistics but we preferred to select a single representative program rather than a large set of non-representative programs.

\subsection{Our protocol to evaluate $p_{s}$}

Our protocol to evaluate $p_{s}$ is based on a set of faulty versions of the specimen program $P$ that are automatically created by a mutation analysis scheme [28]. A mutant $Q$ is a version of $P$ where a single syntactical change has been introduced. Classically, a mutant is said to be killed by a test datum $x$ when $Q(x) \neq P(x)$. In our framework, we will consider a mutant to be killed if there exists $\theta \in S_{n}$ such as $(x, \theta)$ is a symmetry violation for $Q$ w.r.t. $\Psi_{n, m}$. The value of $p_{s}$ depends on $s$, the size of the subdomain of this input space that is considered for the random test data generation. Given a size $s$, the empirical protocol is as follows:

1. built $\left\{Q_{i}\right\}_{i=1 . . K}$ a set of $K$ mutants of a specimen program $P$ that has to satisfy a symmetry relation $\Psi_{n, m}$;

2. for each $Q_{i}$, compute the booleans $b_{i, x}=\left(Q_{i}(\tau \cdot x) \neq \Psi_{n, m}(\tau) \cdot Q_{i}(x) \operatorname{or} Q_{i}(\sigma \cdot x) \neq\right.$ $\left.\Psi_{n, m}(\sigma) \cdot Q_{i}(x)\right)$ for each test datum $x$ of the input domain of $P$; 
3. returns $p_{s}=\frac{1}{N * K} \cdot \sum b_{i, x}$ which is just the median value of the probability for the $K$ mutants.

Note that the programs $Q_{i}$ are executed on a large part of their input domain, hence it is important to select a specimen program having an input space of reasonable size. Note also that only two permutations are required to be checked in this protocol $\left(\tau=\left(\begin{array}{ll}1 & 2\end{array}\right)\right.$ and $\left.\sigma=\left(\begin{array}{lll}1 & 2 & . .\end{array}\right)\right)$. This is a direct consequence of Theorem 1 .

We selected the well-known triangle classification program trityp [12], that belongs to the Software Testing folklore. It takes three non-negative bytes as arguments that represent the relative lengths of the sides of a triangle and classifies the triangle as scalene, isocele, equilateral or illegal. The results of trityp must be invariant to every permutation of its three input values, leading to a $\Psi_{3,1}$ symmetry relation. This program appears to be an interesting specimen candidate as it contains a lot of decisions and the probability of a symmetry violation to occur is highly related to the flow of control. Hence, this probability highly depends on the input subdomain that is being explored. This property has recently been investigated from an experimental point of view in [6].

In our empirical protocol, application of the tool MuJava [28] has lead to build automatically 36 mutants where an arithmetic operator was replaced (AOR), 85 mutants where a relational operator was replaced (ROR), and 14 mutants where a Logical connector was replaced (LCR). In the current MuJava framework, equivalents mutants ${ }^{5}$ are not removed from the set of mutants, although they cannot be revealed by the means of testing [12]. So, 135 mutants of the trityp programs were built by the tool and the input domain was restricted to contain at most $s=126^{3}=2000376$ input values. Among the 135 mutants, 21 were not killed by Symmetric Testing but we kept them in the experiments to avoid introducting a bias in the study.

For each mutant, we compute the number of symmetry violations found when exploring exhaustively a subdomain of the input domain. The average number of symmetry violations that were detected when exploring a subdomain of size $s$ allows for calculating the probability of a symmetry violation to occur by using a uniform random test data generator $\left(p_{s}\right)$. Fig. 2 contains the results we got for several increasing values of $s\left(1^{3}, 6^{3}, 11^{3}, \ldots, 126^{3}\right)$ by distinguishing the class of considered mutants (AOR,ROR,LCR). We compute $p_{s}$ as the center of mass of the 3 bottom values obtained for the greatest size $s\left(s=126^{3}\right)$. Hence, $p_{s}=(36 * 0.057+85 * 0.177+14 * 0.062) / 135=0.133$.

\subsection{Test quality based on symmetry violations}

Based on Theorem 2, we get the model $\left(1-p_{s}\right)^{N}=1-q_{N}$ for random testing based on symmetry checking. The test quality $q_{N}$ differs from $Q_{N}$ because $q_{N}$ is based on symmetry checking and on the size of the input domain. In fact, it represents the probability of $N$ randomly generated test data in a subdomain of size $s$ to reveal at least one symmetry violation in $P$ by the means of Symmetric Testing. When the property is enforced ( $P$ has

\footnotetext{
${ }^{5}$ programs which compute the same outcome as the original program although a mutation operator is applied
}

$\mathrm{RR} \mathrm{n}^{\circ} 5675$ 


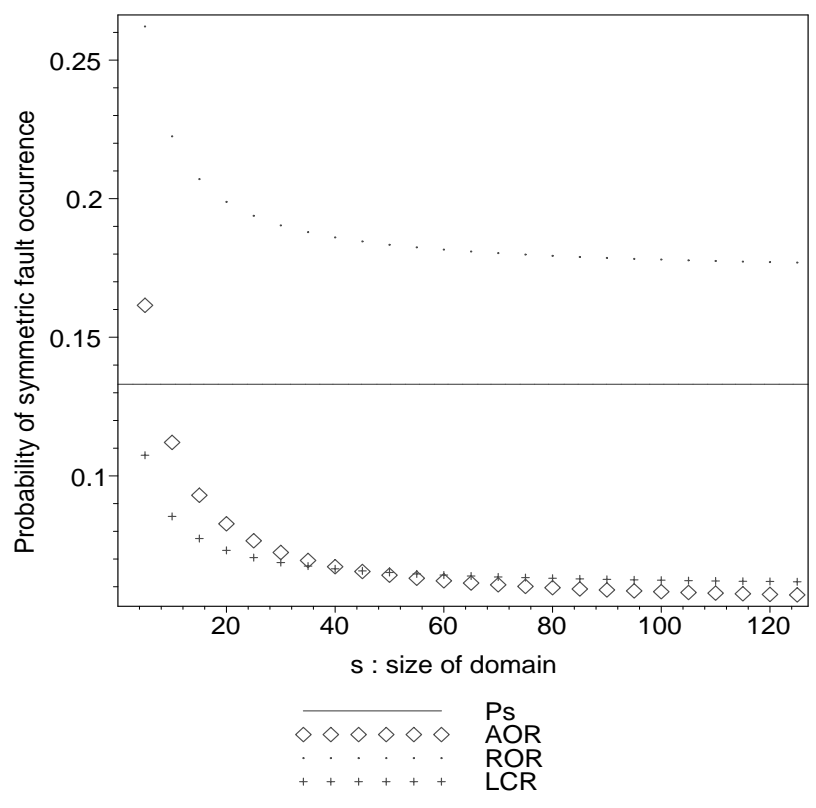

Figure 2: Empirical evaluation of $p_{s}$

been tested with a test quality $q_{N}$ ), we have that the symmetry relation is satisfied by the program $P$ with a probability $q_{N}$. So, by using this model it becomes possible to assess the test quality we obtain by applying Symmetric Testing on $P$.

The test quality was required to be equal to 0.9995 as is usually the case in other works [31]. By using the empirical value of $p_{s}=0.133$ and the corollary $N \geq\left\lfloor\frac{\ln \left(1-q_{N}\right)}{\ln \left(1-p_{s}\right)}\right\rfloor$, we get $N=54$ as a minimum number of randomly generated test data to apply to a program under test. Note that this value of the test quality does not ensure the symmetry relation to be satisfied by $P$ because the probability of a symmetry violation to occur is not zero.

\section{Experimental environment}

The goal of the experiments was to study the applicability of Symmetric Testing to reveal faults within Java Card APIs. The validation process of Java Card APIs is usually made of three distinct phases: firstly, Java card test applets are developed on a host machine by using simulation libraries; secondly, the tests are applied to an emulation code that runs on a card emulator; and finally, the test execution is conducted by cross-testing on the Java card. Our experiments were performed in situations as close as possible to the real usage. Hence, test execution and symmetries checking have been conducted by cross-testing on the 
Java card with the help of a card reader. In this respect, we differ from other smart cards testing research approaches that focus only on test cases generation [9, 29].

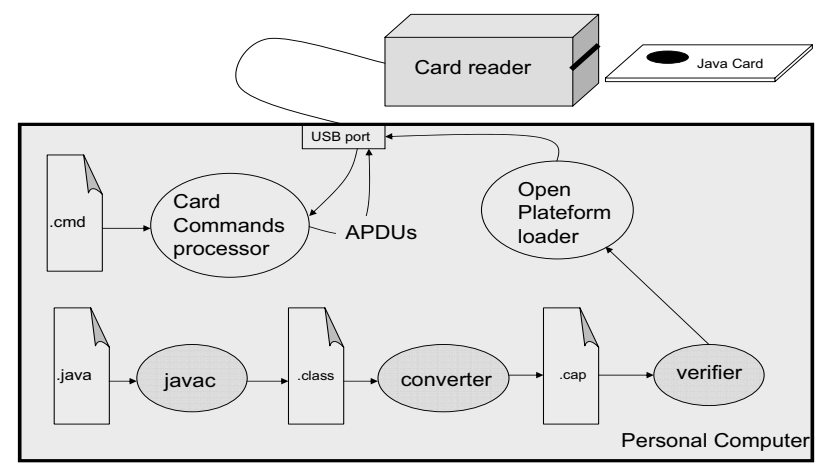

Figure 3: Experimental environment

Fig.3 contains a view of our experimental environment. It is composed of five components: the java compiler (SUN SDK 1.4), the OCS converter that produces standard Java Card byte code (converted applet file), the OCS verifier which statically determines whether a cap file comply with the Java Card specifications, the Open Platform loader which downloads and manages the applets onto the card and a Card Command Processor that sends commands to the smart card via a card-reader interface. Note that the bytecode verification process is done off-card by the OCS verifier. The Card Command Processor is a command interpreter that accepts several language constructs such as conditional and loop.

\subsection{Tests generation and execution}

In our experimental environment, a special attention has been paid to minimize the communications between the reader and the card. Recalling that our goal was to realize the test execution and the symmetry checking processes on-card, passing large sets of random numbers through the APDU mechanism would have been too time-consuming. Hence, we have designed a Java Card applet (to be loaded on-card) that generates test data and that checks the symmetry relations. This applet serves as a test harness and its size is around 1.2 kbytes. It defines a single command TEST_API that launches three executions of the API method under test $(P(x), P(\tau \cdot x), P(\sigma \cdot x))$ and checks the computed output with regards to a given symmetry relation. The applet makes use of a uniform random test data generator provided by the Cosmo API implementation of the javacard. security. RandomData class to generate a single test datum $x$. In case of symmetry violation, a boolean is returned through the APDU mechanism to inform the tester. After having compiled, converted and 
verified the applet, it is loaded on-card by the OP loader. Then, the command TEST_API is launched $N=54$ times with the help of a command script, interpreted by the Card Command processor.

\subsection{Experimental results}

For our experiments, we selected the seven methods from the Cosmo Java Card API Utilfs that have a symmetry relation to satisfy. In the industrial validation process of the Cosmo kit, these methods are systematically tested by using a few values. For instance, the arrayAndNonAtomic method is tested with two randomly generated byte arrays by varying the values of destOff, srcOff and len. By using the approach presented in the paper, we tested each method of the API Utilfs (that has to satisfy a symmetry relation) with 54 randomly generated test data ${ }^{6}$. Tab. 2 contains the time elapsed to pass all the 54 tests for each method. This time value corresponds to the absolute user time (including garbage collections, operating system calls, etc.) elapsed on the 8-bit CPU Cosmo processor. It is just given here to illustrate the interest of using symmetry relations as automatic (partial) test oracles. This time should be compared to the time required by the tester to predict the expected results of the methods with each of the 54 randomly generated test data.

Table 2: Symmetry in the OCS Utilfs methods

\begin{tabular}{|c|c|}
\hline Java Card methods & User time elapsed \\
\hline $\begin{array}{l}\text { short arrayAndNonAtomic ( byte[] dest, short dest0ff, byte[] src, short src0ff, } \\
\text { short len) }\end{array}$ & $13 \mathrm{~min} 59 \mathrm{sec}$ \\
\hline short arrayCompare ( byte[] src, short src0ff, byte patByte, short length) & $6 \mathrm{~min} 15 \mathrm{sec}$ \\
\hline short arrayFindByte ( byte[] src, short src0ff, short len, byte pattern) & $4 \mathrm{~min} 24 \mathrm{sec}$ \\
\hline $\begin{array}{l}\text { short array0rNonAtomic ( byte[] dest, short dest0ff, byte[] src, short src0ff, } \\
\text { short length) }\end{array}$ & $13 \mathrm{~min} 57 \mathrm{sec}$ \\
\hline $\begin{array}{l}\text { short arrayXorNonAtomic( byte[] dest, short dest0ff, byte[] src, short src0ff, } \\
\text { short length) }\end{array}$ & $13 \mathrm{~min} 55 \mathrm{sec}$ \\
\hline $\begin{array}{l}\text { short get0bjectIndex( java.lang.0bject [] src, short src0ff, short n, } \\
\text { java.lang.0bject pattern) }\end{array}$ & $2 \min 01 \mathrm{sec}$ \\
\hline short getShortIndex ( short [] src, short src0ff, short $n$, short pattern) & $2 \min 05 \mathrm{sec}$ \\
\hline
\end{tabular}

The test quality achieved by these tests is equals to 0.9995 , that is to say each of these methods satisfies its symmetry relation with a test quality of 0.9995 . We did not find any symmetry violations during this testing process but this does not prove the absence of symmetry violations as our approach is only probabilistic.

\subsection{Discussion and further work}

The main limitation of the Symmetric Testing paradigm comes from the fact that it cannot be applied to methods that do not have to satisfy a symmetry relation [16]. To address this problem, we plan to use other kinds of properties to check the output correctness of Java Card APIs. Recently, Chen et al. proposed in [7] to use existing relations over the input data and

\footnotetext{
${ }^{6}$ All the randomly generated arrays are of size $0 \times 7 F$ which is the greatest byte value
} 
the computed outcomes to eliminate faulty programs. Formally speaking, let $\left\{I_{1}, . ., I_{n}\right\}_{n>1}$ be $n$ distinct test data for a program intended to compute a function $f$ and suppose that given a relation $r$ over $\left\{I_{1}, . ., I_{n}\right\}$, the results $f\left(I_{1}\right), . ., f\left(I_{n}\right)$ must satisfy a property $r_{f}$, then we have: $r\left(I_{1}, . ., I_{n}\right) \Longrightarrow r_{f}\left(f\left(I_{1}\right), . ., f\left(I_{n}\right)\right)$. These relations, called metamorphic relations, are more general than symmetry relations. In previous work [17], we proposed to automate the generation of input data that violate a given metamorphic relation, by using Constraint Logic Programming techniques. Specifying such metamorphic relations over the Java Card APIs would be interesting as they could serve as (partial) test oracles for non-symmetric methods. Another trail would be to consider formally specified postconditions as a way to check the output correctness. For example, the formal specification of Java Card APIs written in JML (Java Modeling Language) by Poll et al. [25] could be an interesting way of getting formulas that can serve as (partial) test oracle. However, combining these formal postconditions with a random test data generator remain a non-trivial task as they make use of specific constructs that limit the possibility to asses test quality. For example, the formal JML postcondition of the arrayCopy Java Card API method extracted from [5] and shown in Fig. 4 makes use of array accesses and a loop construct for which a fault occurrence probability seems to be difficult to establish.

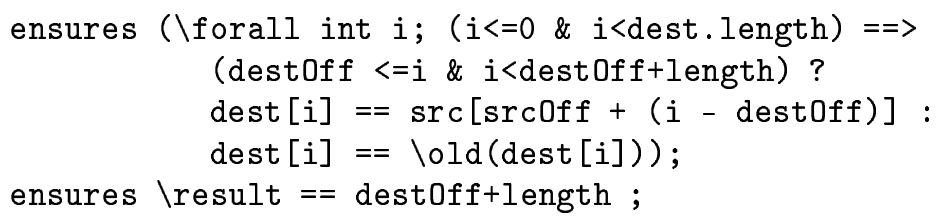

Figure 4: JML postconditions for arrayCopy

\section{Conclusion}

In this paper, we have introduced a software testing framework for on-card testing of symmetric Java Card API methods. The framework checks symmetry relations of methods by using a uniform random test data generator. We have developed a semi-empirical model to help decide when to stop testing and to assess test quality. We have reported on a first experience on testing a few methods of the OCS Cosmo 32 RSA V3.4 Java Card API by using the Symmetric Testing paradigm. The main restriction of this approach comes from its limitation to symmetric methods, that is to say methods that have to satisfy a symmetry relation. Further work includes the exploitation of other kinds of properties to check the output correctness of Java Card methods, such as postconditions extracted from a formal specification of the APIs. Another perspective would be to explore the use of Symmetric Testing to address the resources consumption problem. Due to its limited memory and execution features, Java cards and Java Card APIs must be thoroughly tested w.r.t. memory 
and time consumption. Symmetry relations combined with random testing could be interesting candidates to find counter-examples of statically estimated consumption bounds but finding ways to improve the capacity of random testing to reach this objective remains a difficult challenge.

\section{Acknowledgements}

This work was carried out in part with the support of Amokrane Saibi from Oberthur Card Systems who provided us the Cosmo 32 RSA V3.4 Java Card kit. We would like to thank Thomas Jensen, Franois Hantry, Matthieu Petit and Gerardo Schneider for fruitful discussions on this work.

\section{References}

[1] F. Ambert, F. Bouquet, S. Chemin, S. Guenaud, B. Legeard, F. Peureux, M. Utting, and N. Vacelet. Bz-testing-tools: A tool-set for test generation from $\mathrm{z}$ and $\mathrm{b}$ using constraint logic programming. In In Proc. of FATES'02, Formal App. to Test. of Software, Wkshp of CONCUR'02, pages 105-120, Brnö, Czech Republic, Aug. 2002.

[2] M. A. Armstrong. Groups and Symmetry (Undergraduate Texts in Mathematics). Springer Verlag, second edition, 1988.

[3] Dragan Bosnacki, Dennis Dams, and Leszek Holenderski. Symmetric spin. In SPIN, pages $1-19,2000$.

[4] Jonathan P. Bowen, Kirill Bogdanov, John Clark, Mark Harman, Robert Hierons, and Paul Krause. FORTEST: Formal methods and testing. In Proc. COMPSAC 02: 26th IEEE Annual Int. Computer Software and Applications Conf., Oxford, UK, pages 91101. IEEE Computer Society Press, Aug. 2002.

[5] C.-B. Breunesse, N. Cataño, M. Huisman, and B.P.F. Jacobs. Formal methods for smart cards: an experience report. Science of Computer Programming, 55(1-3):53-80, 2005.

[6] T.Y. Chen, D.H. Huang, T.H. Tse, and Z.Q. Zhou. Case studies on the selection of useful relations in metamorphic testing. In 4 th Ibero-American Symp. on Software Engineering and Knowledge Engineering (JIISIC 04), pages 569-583, 2004.

[7] T.Y. Chen, T.H. Tse, and Zhiquan Zhou. Fault-based testing in the absence of an oracle. In IEEE Int. Comp. Soft. and App. Conf. (COMPSAC), pages 172-178, 2001.

[8] T.Y. Chen, T.H. Tse, and Zhiquan Zhou. Semi-proving: an integrated method based on global symbolic evaluation and metamorphic testing. In ACM Int. Symp. on Soft. Testing and Analysis (ISSTA), pages 191-195, 2002. 
[9] D. Clarke, T. Jéron, V. Rusu, and E. Zinovieva. Automated test and oracle generation for smart-card applications. In In International Conference on Research in Smart Cards (e-Smart'01), Springer Verlag, LNCS 2140, pages 58-70, 2001.

[10] Edmund M. Clarke, Orna Grumberg, Marius Minea, and Doron Peled. State space reduction using partial order techniques. Soft. Tools for Tech. Transfer, 2, 1998.

[11] R. A. DeMillo, W. M. McCracken, R. J. Martin, and J. F. Passafiume. Software Testing and Evaluation. The Benjamin/Cummings Publishing Company, INC., Menlo Park, CA, 1987.

[12] R.A. DeMillo and J.A. Offut. Constraint-based automatic test data generation. IEEE Trans. on Soft. Eng., 17(9):900-910, Sep. 1991.

[13] J.W. Duran and S. Ntafos. An Evaluation of Random Testing. IEEE Trans. on Soft. Eng., 10(4):438-444, Jul. 1984.

[14] F. Allen Emerson and A. Prasad Sistla. Symmetry and model checking. Formal Methods in System Design: An International Journal, 9(1/2):105-131, August 1996.

[15] Patrice Godefroid. Exploiting symmetry when model-checking software. In FORTE, pages $257-275,1999$.

[16] A. Gotlieb. Exploiting symmetries to test programs. In IEEE International Symposium on Software Reliability and Enginering (ISSRE), pages 365-374, Denver, CO, USA, Nov. 2003.

[17] A. Gotlieb and B. Botella. Automated metamorphic testing. In 27th IEEE Annual International Computer Software and Applications Conference (COMPSAC'03), Dallas, TX, USA, November 2003.

[18] U. Hansmann, M.S. Nicklous, T. Schack, and F. Seliger. Smart Card Application Development using Java. Springer Verlag, 2000.

[19] C. Norris Ip and David L. Dill. Better verification through symmetry. Formal Methods in System Design: An International Journal, 9(1/2):41-75, August 1996.

[20] ISO International Standard 15408. Common Criteria for Information Technology Security Evaluation, Aug. 1999. CCIMB-99-033, Part 3: Security assur. req.

[21] B. Legeard and F. Peureux. Generation of functional test sequences from $b$ formal specification : presentation and industrial case study. In In Proc. of ASE'01,International Conference on Automated Software Engineering, IEEE Computer Society Press, pages 377-381, San Diego, USA, Nov. 2001.

[22] B. Legeard, F. Peureux, and M. Utting. Automated boundary testing from $\mathrm{z}$ and $\mathrm{b}$. In In Proc. of FME'02, Formal Methods Europe, Springer Verlag LNCS 2391, pages 21-40, Copenhaguen, Denmark, Jul. 2002.

$\mathrm{RR} \mathrm{n}^{\circ} 5675$ 
[23] Y. Malaiya, M.N. Li, J.M. Bieman, and R. Karcich. Software reliability growth with test coverage. Trans. on Reliability, 51(4):420-426, Dec. 2002.

[24] H. Martin and L.d. Bousquet. Automatic test generation for java-card applets. In Isabelle Attali and Thomas P. Jensen, editors, Java on Smart Cards: Programming and Security, First International Workshop, JavaCard 2000, Cannes, France, September 14, 2000, Revised Papers, volume 2041 of Lecture Notes in Computer Science, pages 121136. Springer, 2001.

[25] H. Meijer and E. Poll. Towards a full formal specification of the java card api. In Isabelle Attali and Thomas P. Jensen, editors, Smart Card Programming and Security, International Conference on Research in Smart Cards, E-smart 2001, Cannes, France, September 19-21, 2001, Proceedings, volume 2140 of Lecture Notes in Computer Science, pages 165-178. Springer, 2001.

[26] S. Ntafos. On Random and Partition Testing. Software Engineering Notes, 23(2):42-48, Mar. 1998.

[27] Oberthur Card Systems. Cosmo 32 RSA V3.4 API Reference Guide, 2003.

[28] Jeff Offutt, Yu-Seung Ma, and Yong-Rae Kwon. An experimental mutation system for java. SIGSOFT Softw. Eng. Notes, 29(5):1-4, 2004.

[29] A. Pretschner, O. Slotosch, H. Lötzbeyer, E. Aiglstorfer, and S. Kriebel. Model based testing for real: The inhouse card case study. In In Proc. 6th Intl. Workshop on Formal Methods for Industrial Critical Systems (FMICS'01), pages 79-94, Paris, Jul. 2001.

[30] SUN Microsystems. Java Card 2.1.1 Application Programming Interface, May 2000.

[31] P. Thévenod-Fosse and H. Waeselynck. An investigation of statistical software testing. Journal of Sotware Testing, Verification and Reliability, 1(2):5-25, July 1991.

[32] E. Weyuker. On testing non-testable programs. The Computer Journal, 25(4), 1982.

[33] T. Wood, K. Miller, and R. E. Noonan. Local exhaustive testing: a software reliability tool. In Proc. of the Southeast regional conf., pages 77-84. ACM Press, 1992. 


\section{Contents}

$\begin{array}{llr}1 & \text { Introduction } & 3\end{array}$

2 Symmetric Testing $\quad 4$

2.1 Symmetry relations ....................... 5

2.2 Examples .......................... 6

2.3 Symmetric Testing . . . . . . . . . . . . . . . . 7

3 Symmetry in Java Card API 8

3.1 The Cosmo Java Card APIs . . . . . . . . . . . . . . . . . . . 8

3.2 Symmetry analysis of a selected Cosmo Java Card API class . . . . . . . . . . 9

4 A semi-empirical model $\quad 11$

4.1 A theoretical model of random testing . . . . . . . . . . . . . . 11

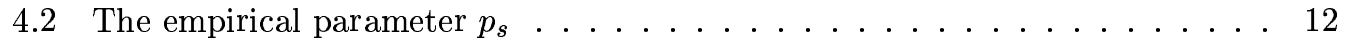

4.3 Our protocol to evaluate $p_{s} \ldots \ldots \ldots \ldots \ldots \ldots \ldots$

4.4 Test quality based on symmetry violations . . . . . . . . . . . . 13

5 Experimental environment 14

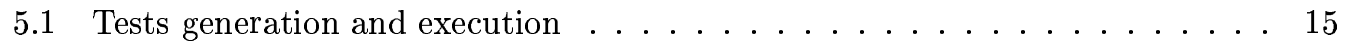

5.2 Experimental results ........................ 16

5.3 Discussion and further work .................. 16

6 Conclusion $\quad 17$

$\mathrm{RR} \mathrm{n}^{\circ} 5675$ 
Unité de recherche INRIA Lorraine, Technopôle de Nancy-Brabois, Campus scientifique, 615 rue du Jardin Botanique, BP 101, 54600 VILLERS LÈS NANCY

Unité de recherche INRIA Rennes, Irisa, Campus universitaire de Beaulieu, 35042 RENNES Cedex Unité de recherche INRIA Rhône-Alpes, 655, avenue de l'Europe, 38330 MONTBONNOT ST MARTIN Unité de recherche INRIA Rocquencourt, Domaine de Voluceau, Rocquencourt, BP 105, 78153 LE CHESNAY Cedex Unité de recherche INRIA Sophia-Antipolis, 2004 route des Lucioles, BP 93, 06902 SOPHIA-ANTIPOLIS Cedex

Éditeur

INRIA, Domaine de Voluceau, Rocquencourt, BP 105, 78153 LE CHESNAY Cedex (France) http://www.inria.fr ISSN 0249-6399 